\title{
Numeralia del subdesarrollo en Zacatecas: el mayor despojo en la región centro-norte
}

Federico Guzmán López*

\begin{abstract}
El objetivo del presente articulo es identificar el comportamiento de los principales indicadores socioeconómicos que dan cuenta de las causas y efectos de la inserción del capital corporativo en el estado de Zacatecas, cuyo impacto ha sido el mayor despojo de riqueza entre las entidades federativas de la región centro-norte de México. La estrategia de mercantilizar el territorio en beneficio de la inversión extranjera directa agrava el extractivismo de materias primas y la explotación laboral con repercusiones en la pobreza, inseguridad y migración.
\end{abstract}

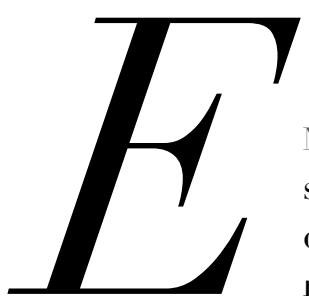

N ESTE TRABAJO analizaremos el subdesarrollo de Zacatecas a partir de información cuantitativa que permita entender la desarticulación de la estructura productiva, como rasgo cualitativo transversal del proceso de extracción y transferencia de materias primas, excedente económico y fuerza de trabajo desde Zacatecas, entidad reducida a economía de enclave para las economías centrales, cuya incidencia genera exclusión social, deterioro de la economía local, depredación del medio ambiente y explotación de la mano de obra barata.

* Estudiante del Doctorado en Estudios del Desarrollo de la Universidad Autónoma de Zacatecas, México.
El concepto central de este texto es el de economía de enclave. Los primeros aportes de dicho concepto ${ }^{1}$ se refieren a la manera de organizar la producción para la extracción y transferencia de riqueza natural y materias primas de la periferia para favorecer el proceso de acumulación de los países centrales. En alusión a la economía de enclave, Perroux señaló que la inversión del capital corporativo se instala en un espacio territorial de manera desvinculada y con bajo efecto multiplicador. ${ }^{2}$

En este artículo entendemos como economía de enclave el proceso a través del cual determinado espacio territorial, principalmente de regiones subdesarrolladas donde se localizan actividades productivas orientadas a la exportación, se realiza sin establecer 
encadenamientos con el mercado local y contribuyen a la valoración del capital corporativo en beneficio de las economías centrales. Dicho proceso permite describir relaciones de dependencia en países subdesarrollados y contribuye a explicar la profundización del desarrollo desigual entre países y regiones.

Para describir la trascendencia de Zacatecas como espacio de instrumentación de la estrategia neoliberal, se tomó como espacio contextual a la región centro-norte del país, también conocida como "El granero de México", que agrupa a los estados de Aguascalientes, Guanajuato, Querétaro, San Luis Potosí y Zacatecas.

Las corporaciones instaladas en la entidad son filiales de las redes globales para ampliar los espacios de valor del capital corporativo (extractivistas, maquiladoras, sucursales bancarias, franquicias, etcétera). La gestión estatal se arropa por la nueva gerencia pública cuya función primordial se encamina a promover a Zacatecas en el exterior y a instrumentar mecanismos de desregulación que faciliten la llegada de inversión de recursos privados del ámbito internacional y nacional. En 2012 se promulgó la Ley para Inversión y el Empleo de Zacatecas que otorga concesiones en materia fiscal con exención de hasta Ioo por ciento en el pago de impuestos y derechos estatales. El agente social está compuesto por organizaciones sociales y económicas que se insertan en la economía local de subsistencia, desarticulados entre sí y con poca incidencia en el desarrollo estatal.
Nueve Causas del subdesarrollo

EN ZaCATECAS

1.- Mercantilización del territorio. La existencia de "2,537 concesiones mineras vigentes al 3i de diciembre de 20го, cubren una superficie de 2' 741,067.5o hectáreas". ${ }^{3}$ De acuerdo con datos de la Secretaría de Economía, ${ }^{4}$ en Zacatecas se obtuvieron 99 concesiones autorizadas en 2012 con una superficie de 260,467 hectáreas. Las cifras anteriores son equivalentes a 4I.66 por ciento de la superficie del territorio zacatecano. La superficie concesionada a la minería en México durante 1993 al 2012 fue 97.86 millones de hectáreas, equivalente al 49.8 por ciento del territorio nacional. ${ }^{5}$

2. - Presupuesto estatal para la modernización neoliberal. El monto del presupuesto estatal refleja una tendencia creciente durante el periodo 2005-20I/4 con 9,165.60 y 24,308.8o millones de pesos, respectivamente; sin embargo la asignación presupuestal por concepto de gasto para 20I4 se orientó, principalmente, a cubrir los pagos a la burocracia estatal, con 38.2 por ciento en el capítulo r,ooo de servicios personales; asimismo se asignó 3o.7 por ciento a transferencias, subsidios y ayudas para mantener los programas clientelares y de control político, y 7.8 por ciento a inversión pública del estado que privilegia las obras de infraestructura y servicios básicos para beneficio del capital corporativo, así como para la militarización de la entidad (ver gráfica i).

Gráfica i

Inversión extranjera directa en Zacatecas, años 1989 al 2013.

(Millones de dólares)

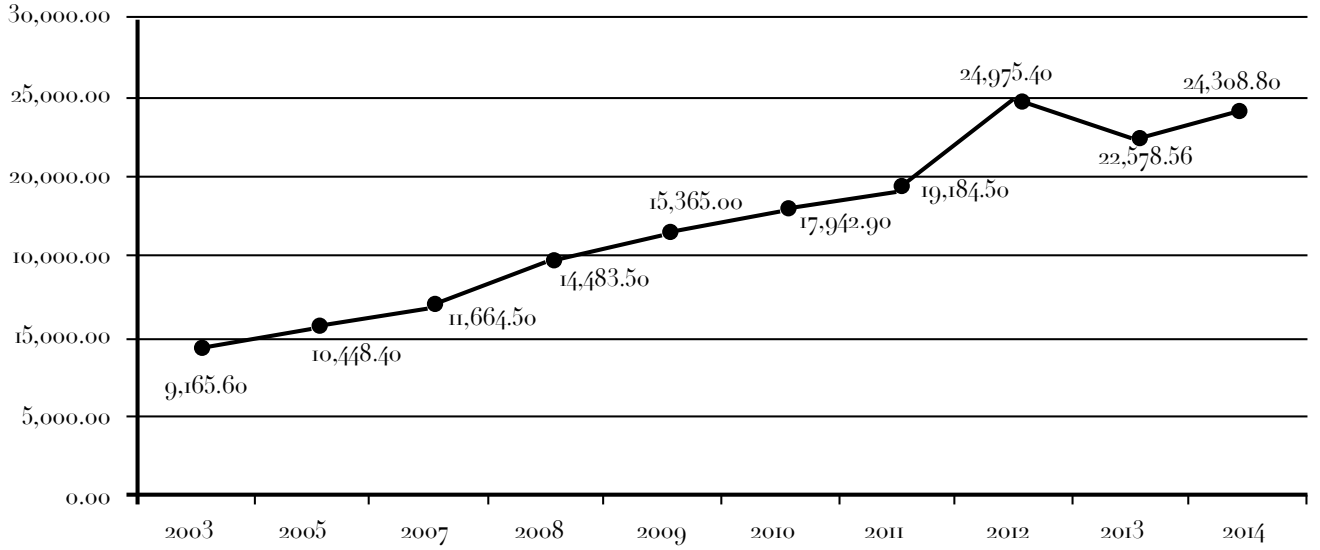

Fuente: Basado en Secretaría de Economía, 1998, 2012, 2013 


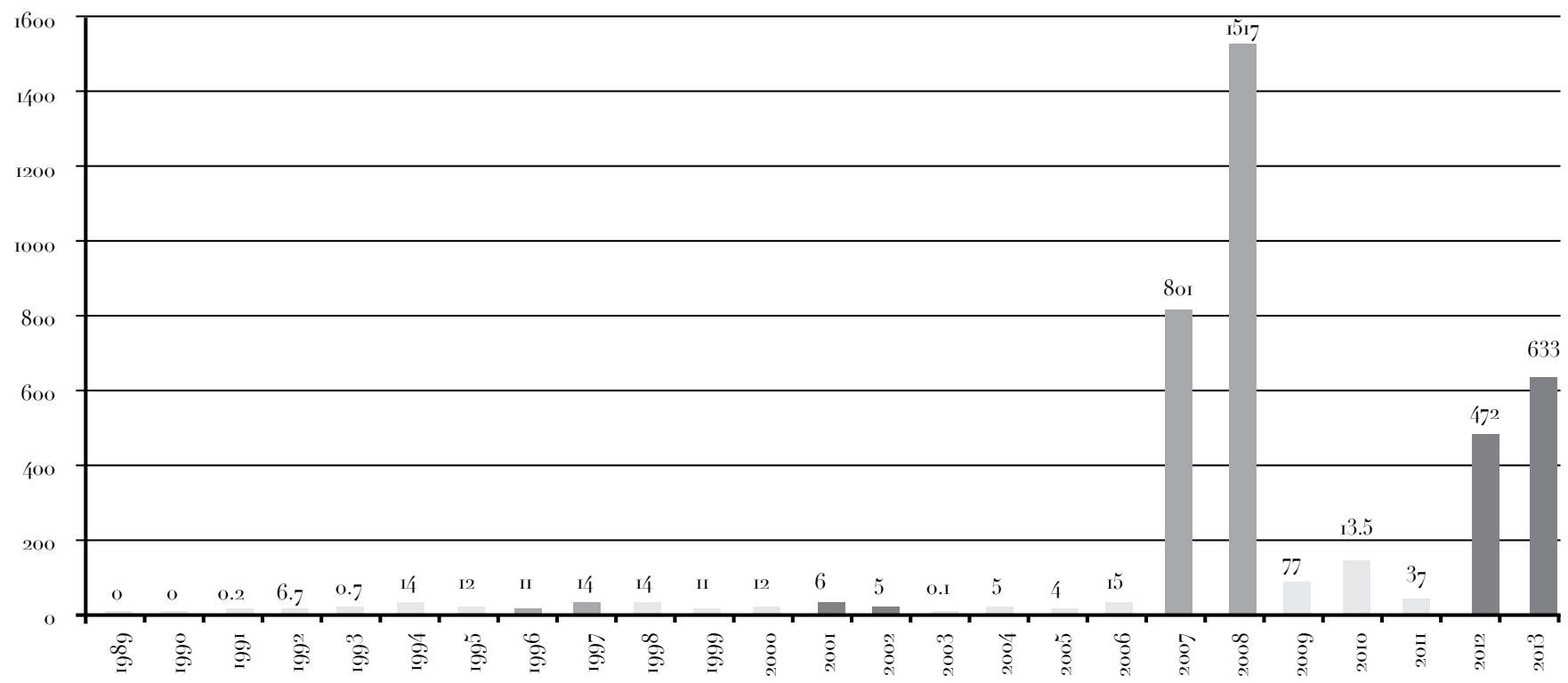

Fuente: Presupuesto de egresos del Estado de Zacatecas, publicado en el Periódico Oficial del Gobierno del Estado de Zacatecas, en los años 2005 al 2014

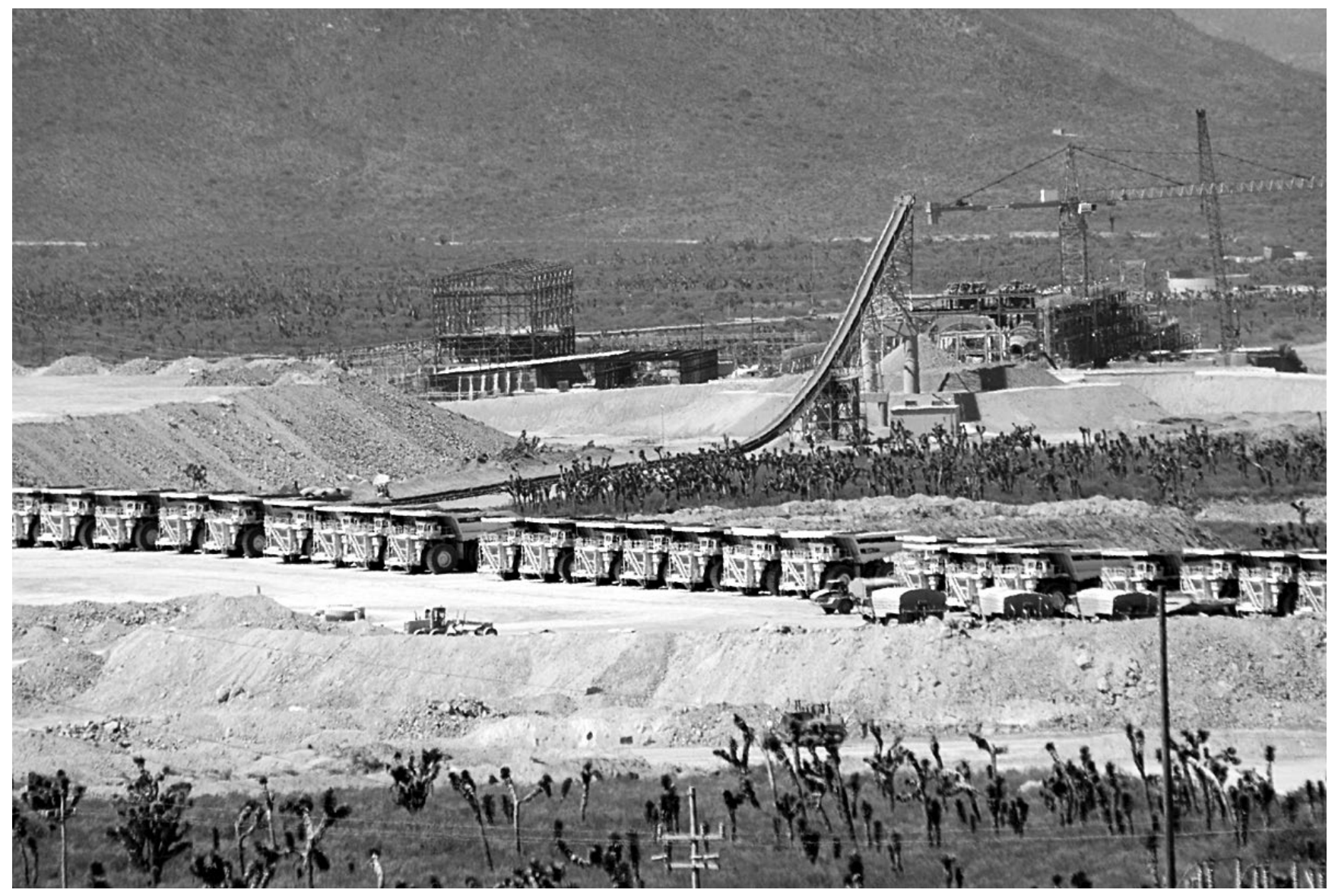

Paro general en minera Peñasquito, Mazapil, Zacatecas, en abril de 2009. 
3. - Inversión extranjera directa. La vocación gerencial del Estado se observa en que de acuerdo a datos de la Secretaría de Economía, ${ }^{6}$ Zacatecas recibió un monto de inversión extranjera directa (IED) en el periodo ı989-2013 de 3,802.2 millones de dólares, equivalente a I.I por ciento a nivel nacional que colocó a Zacatecas en el lugar número $3^{\circ}$ y en $2^{\circ}$ lugar dentro de la región centro-norte, mientras que Querétaro, Guanajuato, Aguascalientes y San Luis Potosí captaron r.3 por ciento, r.r por ciento, I.o por ciento y 0.7 por ciento a nivel nacional, respectivamente. Sólo en 2007, 2008, 2010, 2012 y 20ı3, con montos de captación de 8ог, г,5̆ 7, I35, 472 y 633 millones de dólares, respectivamente, la IED se puede explicar como fuerza motriz de la economía local (ver la gráfica 2).

4.- Extractivismo de materias primas generado en la minería. Durante 2005, el estado de Zacatecas apenas aportaba I/4.9 por ciento de la producción minera nacional,mientras queen 2or2obtuvo 62,962,079.38 miles de pesos, cifra equivalente a 24.34 por ciento del país, colocando a la entidad en el segundo lugar nacional solamente después de Sonora, que produjo 27.42 por ciento en el mismo año. ${ }^{7}$ Para dimensionar lo lucrativo de la minería en el ámbito local de Zacatecas, el valor de la producción para el ejercicio fiscal 20I alcanzó 4,600 millones de dólares $;{ }^{8}$ cifra que equivale a casi tres veces el presupuesto estatal de la entidad para el mismo año por un monto de 19,184.5 millones de pesos.

5. - Extracción de granos y alimentos. La extracción de granos e insumos alimenticios generados en la agricultura, ganadería, aprovechamiento forestal, pesca y caza, cuyo PIB del estado de Zacatecas para 20 оо contaba con ı, 359,736 miles de pesos que representaban 2.3I por ciento de los 448,oI2,I05 miles de pesos del total nacional, colocó a Zacatecas en el lugar $17^{\circ}$ entre los que más aportan al PIB nacional en dicho sector y en el $2^{\circ}$ lugar con la mayor aportación de la región centro-norte, debido a que Guanajuato, San Luis Potosí, Querétaro y Aguascalientes obtuvieron 4.66 por ciento, 2.2I por ciento, I.39 por ciento y I.34 por ciento, respectivamente. 9

6. - Asentamientos comerciales y de servicios. Los asentamientos comerciales y de servicios del estado de Zacatecas para 2010 contaban con $1 / 4553,282$ miles de pesos que representaban el 0.72 por ciento de los 2'014,394,347 miles de pesos del total nacional, ubicando a la entidad en el último lugar, con la menor aportación de la región centro-norte, debido a que Guanajuato, Querétaro, San Luis Potosí y Aguascalientes, aportaron 3.8I por ciento, 2.I5 por ciento, r.69 por ciento y 0.99 por ciento, respectivamente. ${ }^{\circ}$

7.- Explotación de trabajo barato a través de la maquilización automotriz. Ante el vacío de la industrialización endógena, se ha dado impulso a la economía exógena en la industria. En dicho rubro destaca la presencia de maquiladoras de autopartes Delphi Cableados, Yusa y Ahresty. La importancia económica de la industria manufacturera que absorbe a la maquiladora automotriz, para 2010 alcanzó un monto total de i3,6II, gog miles de pesos que representaban o.6o por ciento de los 2,269,3Іо,054 miles de pesos del total nacional que corresponde a industrias manufactureras, ubicándose en el $6^{\circ}$ lugar de las entidades federativas que menos aportan al PIB de la industria manufacturera a nivel nacional. Asimismo, Zacatecas obtuvo el último lugar con la menor aportación de la región centro-norte del país debido a que Guanajuato, San Luis Potosí, Querétaro y Aguascalientes aportaron con 6.84 por ciento, 2.67 por ciento, 2.57 por ciento y г. 86 por ciento, respectivamente."

8. - Turismo cultural. De las diez ciudades mexicanas patrimonio mundial que estableció la Organización de las Naciones Unidas para la Educación, la Ciencia y la Cultura (UNESCO, por sus siglas en inglés), tres se ubican en la región centro-norte del país: Zacatecas, al igual que las ciudades de Guanajuato y Querétaro, forman parte de los espacios urbanos donde se ha mercantilizado el patrimonio cultural. La derrama económica del turismo en Zacatecas contaba con 2393,534 miles de pesos que representaban o.84 por ciento de los $283^{\prime} 425,026$ miles de pesos del total nacional que incluye servicios de alojamiento temporal y de preparación de alimentos y bebidas; ubicándose en el penúltimo lugar con la menor aportación de la región centro-norte, debido a que Guanajuato, Querétaro, San Luis Potosí y Aguascalientes, aportaron 3.2г por ciento, r.69 por ciento, I.57 por ciento y 0.74 por ciento, respectivamente. ${ }^{12}$

9. - Participación social en asuntos públicos. Por el hartazgo de la población y la falta de credibilidad hacia las instituciones del Estado, en promedio registraron una participación de 56.65 por ciento en los últimos cinco procesos electorales celebrados en el estado de Zacatecas ${ }^{13}$ y un nivel de abstencionismo de 43.35 por ciento de los ciudadanos que tomaron la decisión de no votar. ${ }^{14}$ 


\section{Contradicciones de LA MODERNIZACIÓN} NEOLIBERAL EN ZaCatecas.

I.- Pobreza, marginación socialy precarización de la vida familiar. Para 20ıo, Zacatecas, de acuerdo con información del Consejo Nacional de Evaluación de la Política de Desarrollo Social (Coneval), "se ubicó dentro de las ıo entidades con mayor pobreza en el país". ${ }^{55}$ Asimismo, para 2008 había 740 mil 3 oo zacatecanos en situación de pobreza, equivalente a 5o.4 por ciento de la entidad, en 2oro incrementó a 9гі.5 mil, equivalente a 60.2 por ciento y en 2012 disminuyó a 835.5 mil equivalente a 54.2 por ciento. ${ }^{16}$ Para 20ro, el índice de marginación social de Zacatecas es de o.Io4y la entidad contaba con grado de marginación medio, ubicado en el lugar 13 entre las entidades federativas más marginadas a nivel nacional. ${ }^{17}$ En la región centro-norte, Zacatecas se ubica en el segundo lugar de mayor marginación social, debido a que San Luis Potosí es la única entidad de la región que presenta grado de marginación alto; mientras que Guanajuato y
Querétaro cuentan con grado de marginación medio y Aguascalientes con grado de marginación bajo, con o.564, o.o6r, -o.264, -o.9II de grado de marginación, respectivamente. ${ }^{18}$

En relación al Índice de Desarrollo Humano del estado de Zacatecas, de acuerdo con datos de la medición tradicional del periodo 2000-20I0 realizada por la Oficina PNUD México, para 2000 obtuvo un o.76ı5, mientras que para 20 Io alcanzó o.8ı23; dicho valor ubica a la entidad en el primer lugar de más bajo índice de la región centro-norte debido a que los estados de Aguascalientes, Querétaro, San Luis Potosí y Guanajuato obtuvieron o.8529, o.8436, o.8I48 y $0.8 \mathrm{I} 42$, respectivamente.

2.- Subdesarrollo económico local. Para 20ı, el estado de Zacatecas contaba con un PIB de II4,938,669 miles de pesos, que representa $0.9^{2}$ por ciento de los ı2 $508,983,8$ I 5 miles de pesos del total nacional, a precios corrientes, ubicándose en el último lugar, con la menor aportación de la región centro-norte, debido a que Guanajuato, San Luis Potosí, Querétaro y Aguascalientes obtuvieron 3.92 por ciento,

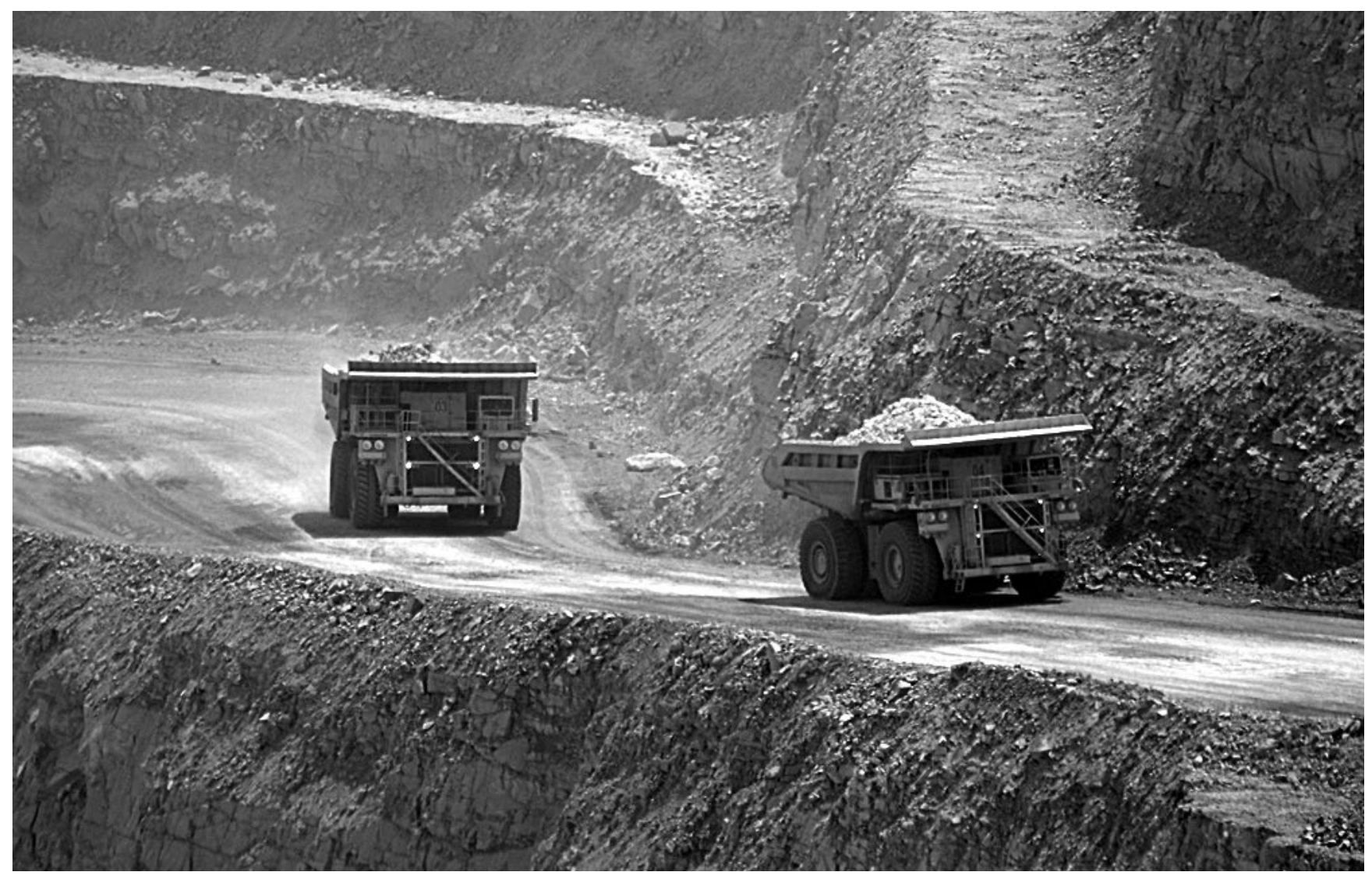




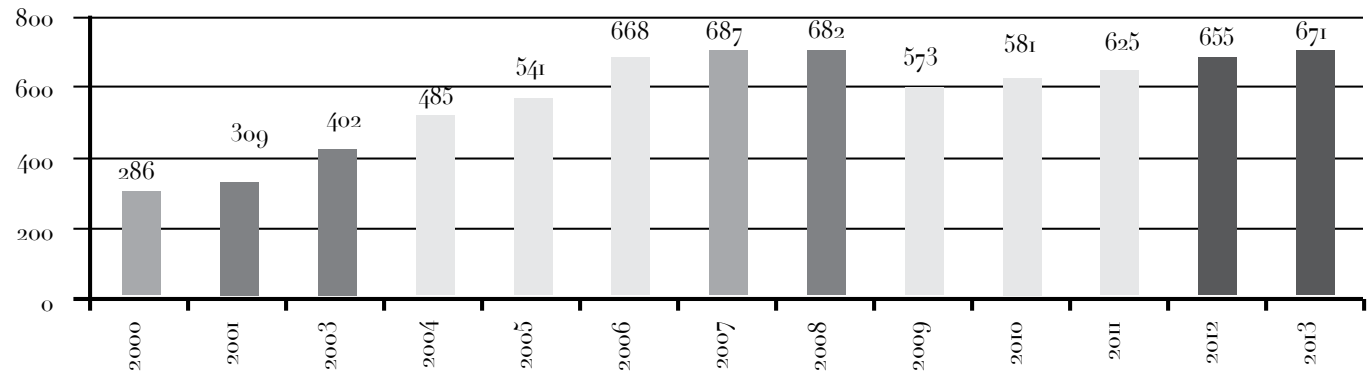

Fuente: Basado en Banco Mundial 2001 y Banxico, 2002, 2013.

I. 87 por ciento, I. 85 por ciento y i.o8 por ciento, respectivamente. ${ }^{19}$ Asimismo, la entidad se ubica entre las cinco entidades que menos valor aportan al PIB nacional, mientras que el promedio nacional es de 3.I2 por ciento. ${ }^{\circ 0}$ La estrategia de desarrollo económico instrumentada por el gobierno estatal ha sido insuficiente para que Zacatecas logre aportaciones superiores a i por ciento del PIB nacional; por ejemplo, para 2006, 2007, 2008, 2009 y 2010, únicamente se alcanzó $0.74,0.74,0.77,0.87$ y $0.9^{2}$ por ciento, respectivamente.

3.- Desempleo estructural. Se registró una tasa de desocupación de 5.4 por ciento, mientras que el año previo, 2oII, fue de 6.5 por ciento; ${ }^{21}$ de acuerdo con la Secretaría del Trabajo y Previsión Social (sTyPS), para el segundo trimestre de 2013 la población económicamente activa eran 6o3,955 personas, 574,528 ocupados y 29,427 desocupados. Sobre el desempleo, Raúl Delgado señaló que "la insuficiencia de fuentes de empleo es cada vez mayor y continúa figurando como el principal problema económico y social de la entidad". ${ }^{22}$

4.- Inseguridad humana y violencia. La inseguridad humana se hace presente en Zacatecas a través del rezago educativo con 500 mil estudiantes que no concluyeron la primaria o secundaria en la entidad. Según Coneval, 286,478 zacatecanos padecían pobreza alimentaria en 2005; asimismo, para 2010, según la Secretaría de Desarrollo Social (Sedesol), en Zacatecas 20.9 por ciento de los habitantes padecieron pobreza alimentaria. Además la ausencia de oportunidades para la población juvenil de i2 a 29 años de edad abarca a 120 mil jóvenes "ninis" zacatecanos, que ni estudian ni trabajan.

En cuanto a la problemática de las violencias, se sigue la directriz nacional de combate al crimen or- ganizado. Las principales obras de infraestructura se basan en la construcción de tres bases militares en los municipios de Fresnillo, Jalpa y Villa Hidalgo.

5. Emigración y despoblamiento. La migración internacional es la dominante, para 2003 y 20 oro el estado de Zacatecas ocupó el primer lugar nacional en el Índice de Intensidad Migratoria con 2.58 y 4.42, respectivamente, y en el monto de remesas per cápita con 340 y 388 dólares, respectivamente; mientras que en la región centro-norte los estados de Guanajuato, San Luis Potosí, Aguascalientes y Querétaro alcanzaron un 3.89, 2.66, 2.49, y 2.25, respectivamente, en cuanto al índice mencionado. Respecto a la migración a Estados Unidos, "la población de origen zacatecano residente en aquel país era de i.2 millones en 2000 y de i.5 millones en $2010 " ;{ }^{23}$ la cifra anterior correspondiente a 2010 equivale a I02 por ciento de la población del estado de Zacatecas.

Basado en datos del Banco Mundial para 2000 y de acuerdo al Banco de México ${ }^{24}$ de 200I a 20I3, Zacatecas generó un monto acumulado de 7,165 millones de dólares de ingresos por remesas familiares (ver gráfica 3). Zacatecas se ubicó en el lugar i $3^{\circ}$ a nivel nacional entre las entidades que captaron mayor monto de remesas en dicho periodo; mientras que en la región centro-norte ocupó el lugar $2^{\circ}$ debido a que los estados de Guanajuato, San Luis Potosí, Querétaro y Aguascalientes captaron 22,327 millones de dólares, y 7,II7 millones de dólares, 4,277 millones de dólares y 3,56o millones de dólares, respectivamente. ${ }^{25}$

Durante las últimas seis décadas Zacatecas ha registrado la tasa más baja de crecimiento poblacional a nivel nacional, en promedio la mitad de la tendencia nacional. Para los años de I98o, I990, 
2000 y 20I0, la entidad contaba con una población

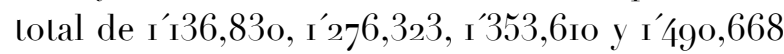
habitantes, respectivamente. ${ }^{26}$ Dichas cifras permitieron que durante el periodo mencionado se obtuvieran tasas de crecimiento demográfico positivas en Zacatecas. Sin embargo, para ı980, ı99o, 2000 y 2005, un total de I6, 2I, 34 y 4 I municipios, respectivamente, registraron una tasa negativa de crecimiento, es decir, despoblamiento.

6. - Vulneración de ecosistemas. Investigaciones del Instituto Nacional de Investigaciones Forestales, Agrícolas y Pecuarias (INIFAP) señalan que: "más de ı5o mil hectáreas de tierras de cultivo del noroeste del estado están en proceso de convertirse en desierto y al menos otras 300 mil padecen erosión severa $[\ldots] \mathrm{Al}$ año se pierden entre 3 o y 40 toneladas de suelo o arcilla por hectárea" ${ }^{27}$

\section{Conclusión}

Las causas del subdesarrollo en Zacatecas, en términos cuantitativos, se reflejan en la dependencia de la dinámica económica del sector primario, con datos del extractivismo de la minería, así como en la producción de granos y alimentos, que contrasta con el rezago que experimentan las actividades industrial y servicios. Otra causa es la asignación del presupuesto estatal con mínima incidencia en la generación de empleos y combate a la pobreza. Los efectos de la modernización neoliberal quedan palpables a través de la estrategia de desarrollo económico con aportaciones inferiores al i por ciento del PIB nacional, en consecuencia la escasez de nuevas fuentes de empleo creadas se ha configurado como el principal problema económico y social. En términos sociales llama la atención indicadores como la consolidación de la pobreza y el segundo de representar el estado más marginado de la región centro-norte de México, con el Índice de Desarrollo Humano más bajo de la región durante 2000-20ıo; además de tener el primer lugar nacional en el índice de intensidad migratoria asociada al incremento de municipios con tasa negativa de crecimiento y despoblamiento.

Los indicadores socioeconómicos presentados reflejan una contradicción: incrementos del presupuesto estatal, la IED, del PIB estatal, valor producción minera y remesas familiares en correlación con aumento de emigración y despoblamiento, así como aumentos y disminuciones de la pobreza. Dicha contradicción ocasiona que Zacatecas sea el estado de la región centro-norte de México con el mayor nivel de subdesarrollo. Resulta indispensable que los agentes del Estado y de la sociedad civil local institucionalicen un proyecto estatal de desarrollo distinto al que ha impuesto el capital corporativo, que permita reorientar la estrategia que ha profundizado el papel de economía de enclave de Zacatecas, que ha beneficiado a economías centrales, que considere la reorientación del presupuesto estatal para fortalecer la economía local de subsistencia y mejore la seguridad humana.

\section{- notas $\cdot-$}

'K. Wilson y A. y Portes (I980), "Immigrant enclaves: an analysis of the labour market exeprience of cuban in Miami", American Journal of Sociology, 84; Maristella Svampa, Lorena Bottaro y Marian Sola (2009), "La problemática de la minería metalífera a cielo abierto: modelo de desarrollo territorio y discurso dominante", en Maristella Svampa y Mirta Antonelli (eds.), Minería trasnacional, narrativas del desarrollo y resistencias sociales, Argentina.

${ }^{2}$ Francois Perroux (1984), El desarrollo y la nueva concepción de la dinámica económica, Barcelona, Serbal-UNesco.

${ }^{3}$ Servicio Geológico Mexicano (20II), Panorama minero del estado de Zacatecas, se, México, p. II.

4Auditoría Superior de la Federación (2012), Auditoría Financiera y de Cumplimiento I2-0-10I00-02-0030, Otorgamiento de Concesiones e Ingresos por Derechos en Materia Minera, Secretaría de Economía, Distrito Federal, México, p. ıo.

${ }^{5}$ Semarnat (2012), Compendio de estadísticas ambientales 2012, México, disponible en:http://dgeiawf.semarnat.gob.mx:8o8o/ibi_
apps/WFservlet?IBIF_ex=D2_energiao5_o2\&IBIC_user=dgeia_ mce\&IBIC_pass=dgeia_mce, $($ Consultado el 2i de febrero de 2013).

${ }^{6}$ Secretaría de Economía (2013), Informe estadístico sobre el comportamiento de la inversión extranjera directa en México, (enerodiciembre de 2013), Comisión Nacional de Inversiones Extranjeras, Distrito Federal, México.

־Secretaría de Economía (2012), IED trimestral por entidad federativa, Distrito Federal, México.

${ }^{8}$ Alfredo Valadez, (La Jornada, 4 de febrero de 2013), Ganancias de mineras en Zacatecas aumentaron casi $300 \%$ en dos años, disponible en p. 27.

9INEGI (2012), Sistema de Cuentas Nacionales de México, Producto Interno Bruto por entidad federativa 2006-2010, México, p. 66.

Ibid., p. $9^{6 .}$

"Ibid., p. 74.

"Ibid., p. пा8.

${ }^{13}$ (IFE, 2OI2).

$14($ IEEZ, 2OI3).

${ }^{15}$ Coneval (2012), Evolución de la pobreza y pobreza extrema nacional y en entidades federativas, 2010-2012, México, р.II. 
${ }^{16}$ Ibid., p. 2012.

${ }^{17}$ Conapo (201I), Índice de marginación por entidad federativa y municipio 2010, Distrito Federal, México, disponible en www. conapo.gob.mx (Consultado el 26 de marzo de 20I4).

${ }^{18}$ Idem.

${ }^{19}$ Basado en datos de (INEGI, 2OI2).

${ }^{20}$ INEGI, op. cit, p. 52.

"Idem.

${ }^{22}$ Raúl Delgado (2000), "Consideraciones sobre la estructura económica y social de Zacatecas de cara al siglo Xxı", en Rodolfo García y Juan Padilla, (coords.) (2000), Los retos demográficos de Zacatecas en el siglo XXI, Secretaría Académica de la UAZ, Zacatecas, México, pp. 26-27.
${ }^{23}$ Humberto Márquez, Raúl Delgado y Rodolfo García (2012), "Modernización neoliberal en Zacatecas: extractivismo, insustentabilidad y migración", en Rodolfo García y Francisco Contreras, (coords.) (2012), Seminario Estatal de Universitarios: por una Nueva Estrategia de Desarrollo Integral para Zacatecas, Unidades Académicas de Economía y en Estudios del Desarrollo, UAZ, Zacatecas, México, p. 37.

4́Banxico (2013), Remesas familiares, México.

25. Idem.

${ }^{26}$ Basado en datos del INEGI.

${ }^{27}$ Humberto Márquez, Raúl Delgado y Rodolfo García (2012), op. cit. p. 24 .

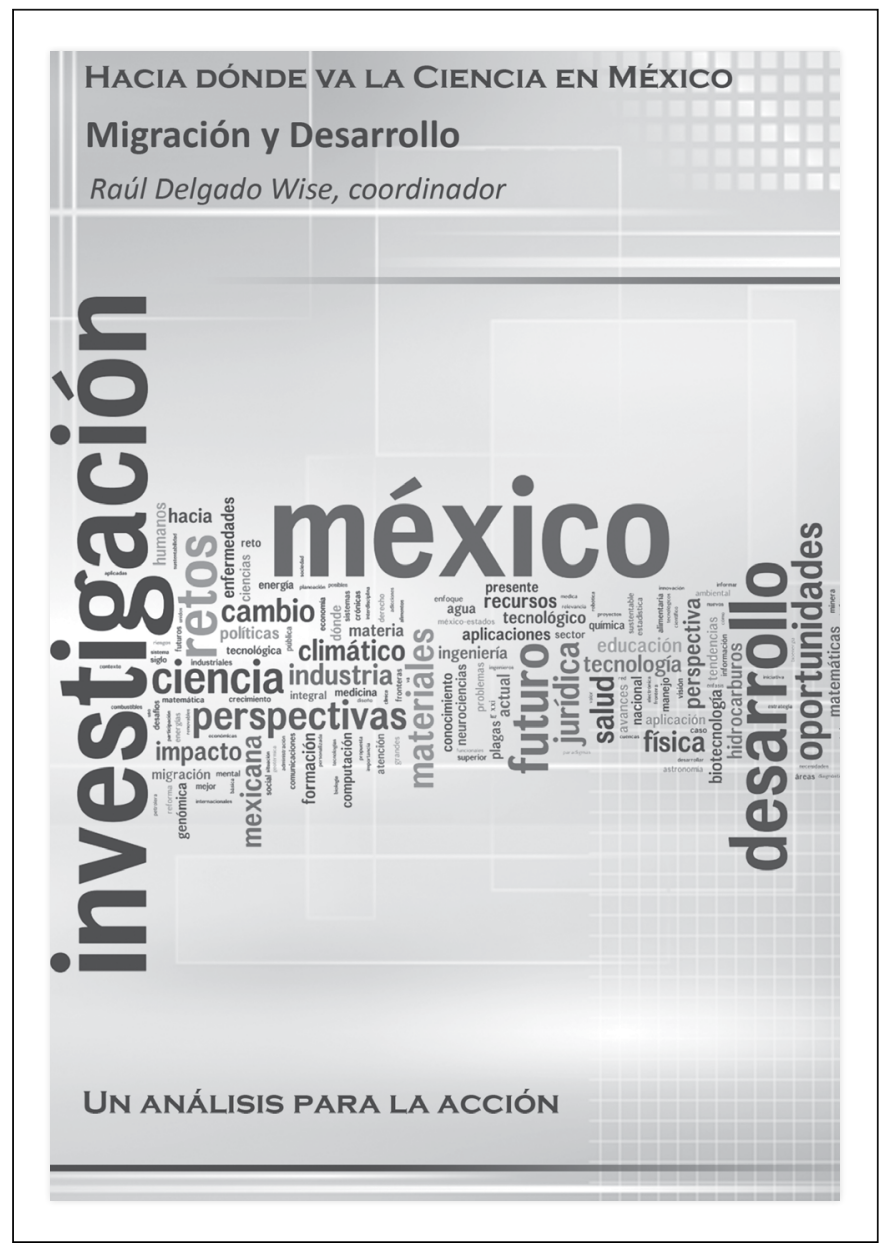

